\title{
Comparative study of different analytical methods for the determination of sterols in human serum by liquid chromatography coupled to mass spectrometry
}

\author{
I. Mendiara, K. Bentayeb, C. Domeño, C. Nerín \\ University Group of Analytical Research (GUIA) \\ Institute of Engineering Research of Aragón (I3A) \\ University of Zaragoza, Mariano Esquillor s/n, 50018, Zaragoza, Spain. \\ Tel.+34-976761873, Fax +34-976762388, e-mail: isabelmn@unizar.es
}

\begin{abstract}
The determination of sterols in human serum allows the diagnosis of inherited disorders in cholesterol metabolism and the evaluation of cardiovascular disease risk. Ten sterols were included in this work: desmosterol and lanosterol (cholesterol precursors); stigmasterol, cholestanol, campesterol, sitosterol and sitostanol (phystosterols) and 7- $\alpha$-hydroxy 4-cholesten3-one, 24-hydroxycholesterol and 27-hydroxycholesterol (oxysterols). Historically, sterols have been analyzed by gas chromatography, which involves laborious and time-consuming derivatization steps. Nowadays, liquid chromatography coupled to mass spectrometry has also been used for the analysis of sterols in serum and mammalian cells and tissues.

The aim of this study is to reduce the sample processing time of the determination of sterols in human serum.Two different approaches have been compared: an off-line and on-line system. Both methods need a suitable cartridge for cleaning the sample before its subsequent chromatography analysis. Solid phase extraction (SPE) cartridges were used for off-line sample treatment. Six different retention mechanisms were studied, including nonpolar, polar and ionic interactions. In the on-line system, the sample treatment was conducted with the help of a restricted access material (RAM). This sorbent represent a special class of materials that are able to fractionate a biological sample into protein and analyte fractions, based on molecular weight cut-off. The limits of quantification obtained in the off-line method were between 8 and $274 \mathrm{ng} / \mathrm{mL}$. In the on-line method limits of detection and quantification found ranged between $0.01 \mathrm{ng} / \mathrm{mL}$ (7- $\alpha$-hydroxy-4-cholesten-3-one) and $0.5 \mu \mathrm{g} / \mathrm{mL}$ (sitostanol) and from $0.03 \mathrm{ng} / \mathrm{mL}$ and $1.7 \mu \mathrm{g} / \mathrm{mL}$ respectively.
\end{abstract}

